\title{
Editorial
}

\section{The difficulties in assessing patients with moderate aortic stenosis}

Recent insights into the relation between haemodynamic severity and clinical outcome have altered our approach to the patient with moderate valvar aortic stenosis. At the heart of this discussion is the changing definition of "severe" (and hence "moderate") aortic stenosis. Historically, severe stenosis was defined in terms of valve area based on the fluid dynamics concept that flow is not restricted until an orifice is reduced to a quarter its original size. As patients typically underwent cardiac catheterisation only after symptom onset, few data on disease progression or haemodynamic severity in asymptomatic patients were available. However, clinical experience supported this definition and it was used successfully for many years to identify patients likely to benefit from valve replacement.

\section{Relation between haemodynamic severity and clinical symptoms}

The first indication that the traditional definition of severe aortic stenosis might need revision came from clinical studies showing substantial overlap in haemodynamic severity between symptomatic and asymptomatic adults despite very different clinical outcomes. ${ }^{1-4}$ While the observed overlap in pressure gradients was not surprising given their flow dependence, the overlap in valve areas (even when indexed for body size) was unexpected, leading to the question: why do some patients become symptomatic with only "moderate" stenosis while others remain asymptomatic despite "severe" valvar obstruction?

The second piece of evidence suggesting that the traditional definition of severe stenosis might no longer be adequate for patient management was the demonstration that not only pressure gradients but also valve areas and other measures of stenosis severity including valve resistance vary with changes in volume flow rate, whether calculated by traditional invasive or newer non-invasive approaches. ${ }^{56}$ The lack of a measure of stenosis severity that is constant for a given valve anatomy raised the question: what criteria should be used to define "severe" aortic stenosis?

\section{New definition of aortic stenosis severity}

If we pause for a moment to consider why we need a definition of severe stenosis, it becomes clear that the purpose of classifying disease severity is to optimise patient management. Thus, the definition of aortic stenosis severity should be based on clinical outcome. In addition, the ideal measure should be accurate, relatively easy to measure, and reproducible.

While there is no single numerical value that defines "severe" aortic stenosis in all patients, clinical outcome data indicate that the initial classification of stenosis severity can be based on the simple Doppler measurement of jet velocity. Jet velocity has the advantages that it requires no calculations, can be obtained in nearly all patients, reflects the transaortic pressure gradient, and has low measurement variability. On the other hand, it must be noted that accurate data recording requires a meticulous examination by a trained and experienced sonographer to avoid under- estimation of stenosis severity. Further, even small differences in velocity may have substantial clinical implications, with these differences being magnified by the squared relation between velocity and pressure gradient.

The following working definitions are proposed as a clinical framework for patient evaluation. Of course, as with other diagnostic tests, Doppler jet velocity cannot be used in isolation but must be integrated with other clinical data in each patient. In addition, further validation and refinement of these proposed definitions will be needed as we gain further understanding of the natural history of aortic stenosis.

In symptomatic adults a jet velocity $>4.0 \mathrm{~m} / \mathrm{s}$ (maximum pressure gradient $>64 \mathrm{~mm} \mathrm{Hg}$, mean gradient $>38 \mathrm{~mm} \mathrm{Hg}$ ) confirms severe obstruction requiring valve replacement. In addition, about $80 \%$ of asymptomatic patients with a jet velocity $>4.0 \mathrm{~m} / \mathrm{s}$ will develop symptoms requiring valve replacement within two years. Thus, based on clinical outcome data, a working definition of severe aortic stenosis is a jet velocity $>4.0 \mathrm{~m} / \mathrm{s}$. Rarely a high velocity is seen with only moderate stenosis in the setting of an increased cardiac output-for example, fever, anaemia or pregnancy. In these patients, valve area should be calculated with re-evaluation after the acute event.

Conversely, in patients with a sclerotic valve and a jet velocity $<3.0 \mathrm{~m} / \mathrm{s}$ (maximum gradient $<36 \mathrm{~mm} \mathrm{Hg}$, mean gradient $<22 \mathrm{~mm} \mathrm{Hg}$ ), symptoms are rarely caused by valvar obstruction, and the likelihood of developing symptoms over the next five years is very low, suggesting these patients can be considered to have mild aortic stenosis. However, exceptions do occur. Severe aortic stenosis occasionally is present with a jet velocity $<3.0 \mathrm{~m} / \mathrm{s}$ if there is a concurrent severe reduction in transaortic volume flow rate-for example, owing to left ventricular systolic dysfunction. In this situation, valve area calculations again are necessary.

\section{Clinical outcome in moderate aortic stenosis}

In asymptomatic patients with moderate aortic stenosis, defined as a jet velocity between 3.0 and $4.0 \mathrm{~m} / \mathrm{s}$, the expected rate of haemodynamic progression is an annual increase in jet velocity of $0.3 \mathrm{~m} / \mathrm{s}$, an annual increase in mean pressure gradient of $7 \mathrm{~mm} \mathrm{Hg}$, and an annual decrease in aortic valve area of $0.1 \mathrm{~cm}^{2}$. The annual rate of symptom onset is approximately $17 \%$ so that by five years of follow up, $85 \%$ of patients have either died or undergone valve replacement. Although early surgical intervention in patients with asymptomatic aortic stenosis has been proposed with a goal of preventing left ventricular hypertrophy and persistent postoperative diastolic dysfunction, ${ }^{7-9}$ most clinicians defer surgical intervention until symptom onset. However, if the patient is undergoing coronary artery bypass grafting, concurrent aortic valve replacement may be considered to avoid early reoperation. As there is a continuum of disease severity within this group, the specific haemodynamic data in each patient should be weighed in this clinical decision.

Clinical decision making in the symptomatic adult with "moderate" aortic stenosis is complex. Although the 
patient appears to have "moderate" aortic stenosis, in fact severe obstruction may be present with a low jet velocity (and transvalvar gradient) if left ventricular systolic dysfunction is present. Calculation of valve area is helpful as a large valve area $\left(>1.0 \mathrm{~cm}^{2}\right)$ indicates that stenosis is not severe, and valve replacement is unlikely to be beneficial. However, if the calculated valve area is $<1.0 \mathrm{~cm}^{2}$, it is unclear if leaflet opening is reduced secondary to impaired ventricular ejection force or if the increased afterload from a severely stenotic valve is affecting ventricular ejection performance.

There are several approaches to this difficult clinical problem. First, the extent of leaflet calcification and thickening can be assessed by echocardiography, including transoesophageal imaging or fluoroscopy if transthoracic images are suboptimal. Significant valve calcification suggests primary valve dysfunction. Second, other causes of left ventricular dysfunction, such as myocardial infarction or cardiomyopathy, should be considered as improvement after valve replacement is less likely if there is intrinsic myocardial dysfunction. Coronary angiography and evaluation for reversible ischaemia certainly may impact the clinical decision making process. However, in the absence of clear cut ischaemia it is difficult to separate intrinsic myocardial dysfunction from impaired ejection performance owing to the increased afterload of aortic stenosis. While evaluation of the relation between the velocity of circumferential wall shortening and end systolic wall stress has been used in the research setting, this approach is rarely practical clinically. Another approach has been to develop alternate measures of stenosis severity, such as valve resistance and stroke work loss. However, there are theoretical problems with these methods and they are not independent predictors of clinical outcome. ${ }^{3}$

Instead, attention has focused recently on assessment of stenosis severity at two different flow rates in adults with aortic stenosis and left ventricular dysfunction. Although flow dependence initially was perceived as a limitation, in fact the change itself may be a more useful marker of disease severity than any single resting value. The extent of leaflet opening depends on both leaflet stiffness and ventricular ejection force; therefore, if the valve remains flexible enough that leaflet opening (and valve area) increases with an increase in flow rate, it is likely that ventricular dysfunction is the primary disease process. However, if valve stiffness exceeds the ability of increased ventricular ejection force to open the leaflets further, the lack of increase in valve area suggests that valve replacement may be beneficial.

Assessment of the change in valve area can be accomplished by calculation of valve area at rest and after exercise, with dobutamine stress echocardiography, or after a period of medical treatment for heart failure. However, caution is needed for several reasons. First, repeated calculation of valve area is technically difficult and the expected physiological changes are close to the reported measurement variability. Second, while there are statistically significant group mean differences, the change in valve area in an individual patient may not reliably predict the response to valve replacement. Third, if there is no change in stroke volume, it remains unclear if the myocardium is unresponsive or if valve area is fixed owing to stiff leaflets. Finally, even patients with left ventricular dysfunction benefit from valve replacement if aortic stenosis is present, with an acceptable mortality and morbidity.$^{10}$ Thus, unless there is convincing evidence that aortic stenosis is not severe in a symptomatic patient with a calcified valve and left ventricular systolic dysfunction, valve replacement should be considered in the clinical decision making process.

CATHERINE M OTTO

Division of Cardiology, Box 356422

University of Washington,

Seattle, WA 98195, USA

1 Turina J, Hess O, Sepulcri F, et al. Spontaneous course of aortic valve disease. Eur Heart $\mathcal{F}$ 1987;8:471-83.

Horstkotte D, Loogen F. The natural history of aortic valve stenosis. Eur Heart F 1988;9(suppl E):57-64.

3 Otto CM, Burwash IG, Legget ME, et al. A prospective study of asymptomatic valvular aortic stenosis: clinical, echocardiographic, and exercise predictors of outcome. Circulation 1997;95:2262-70.

4 Kelly TA, Rothbart RM, Cooper CM, et al. Comparison of outcome of symptomatic to symptomatic patients older that 20 years of age with valvusymptomatic to symptomatic patients older that 20 .

5 Burwash IG, Pearlman AS, Kraft CD, et al. Flow dependence of measures of aortic stenosis severity during exercise. $\mathcal{F}$ Am Coll Cardiol 1994;24:1342-50.

6 Burwash IG, Thomas DD, Sadahiro M, et al. Dependence of Gorlin formula and continuity equation valve areas on transvalvular volume flow rate in valvular aortic stenosis. Circulation 1994;89:827-35.

7 Lund O. Valve replacement for aortic stenosis: the curative potential of early operation. Scand 7 Thorac Cardiovasc Surg 1993;40:1-137.

8 Hess OM, Villari B, Krayenbuehl HP. Diastolic dysfunction in aortic stenosis. Circulation 1993; 87(suppl IV):73-6.

9 Villari B, Vassalli G, Schneider J, et al. Age dependency of left ventricular diastolic function in pressure overload hypertrophy. 7 Am Coll Cardiol 1997;29:181-6.

10 Connolly HM, Oh JK, Orszulak TA, et al. Aortic valve replacement for aortic stenosis with severe left ventricular dysfunction. Prognostic indicators. Circulation 1997;95:2395-400. 